\title{
Physical Activity in an Emirati Cohort: Relationship with Diabetes and Obesity
}

\author{
Alkaf B', Lessan N', Barakat MT', Chambers JC² \\ ' Imperial College London Diabetes Centre, Abu Dhabi, UAE \\ ${ }^{2}$ Imperial College London, London, UK
}

\section{Background}

The increasing prevalence rates of type 2 diabetes mellitus (T2DM) in the Middle East in general, and the UAE in particular, has been attributed to several lifestyle and environmental risk factors, including lack of physical activity. However, the contribution of physical inactivity to weight gain and diabetes in the UAE has been poorly investigated.

\section{Aim}

Investigate the association of physical inactivity with T2DM in a cohort of Emiratis, and determine whether it is independent of body mass index (BMI).

\section{Methods}

(I) Subjects

966 Emiratis recruited from Imperial College London Diabetes Centre (ICLDC)

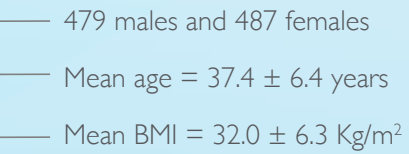

(II) Screening/Diagnosis

\begin{tabular}{|l|l|}
\hline Glycemic status & BMI category \\
\hline Normoglycemia & $\begin{array}{l}\text { Normal } \\
\text { BMI }=18-29 \mathrm{Kg} / \mathrm{m}^{2} \\
\text { Prediabetes }\end{array}$ \\
Obese \\
BMl $=30-39 \mathrm{Kg} / \mathrm{m}^{2}$ \\
T2DM
\end{tabular}

(III) Physical Activity Data:

Global Physical Activity Questionnaire (GPAQ)2

Total physical activity (TPA) in 3 domains; work, transport, and leisure, categorized into:

LPA: Low Physical Activity

MPA: Moderate Physical Activity

HPA: High Physical Activity

\section{Results (I)}

- Prevalence of low physical activity (LPA) was 67.6\%. Rates were similar across participants regardless of diabetes status.

- Prevalence of T2DM was similar among participants with LPA (18.3\%) and those with MPA/HPA combined (16.5\%).

Results (II)

- Similar distribution of physical activity across the 3 domains; work, transport, and leisure among participants irrespective of diabetes status (Figure I).

- Prevalence of T2DM was similar among all participants irrespective of activity levels or BMI group (Figure 2).

- Logistic regression analyses showed no significant association of LPA with T2DM risk.

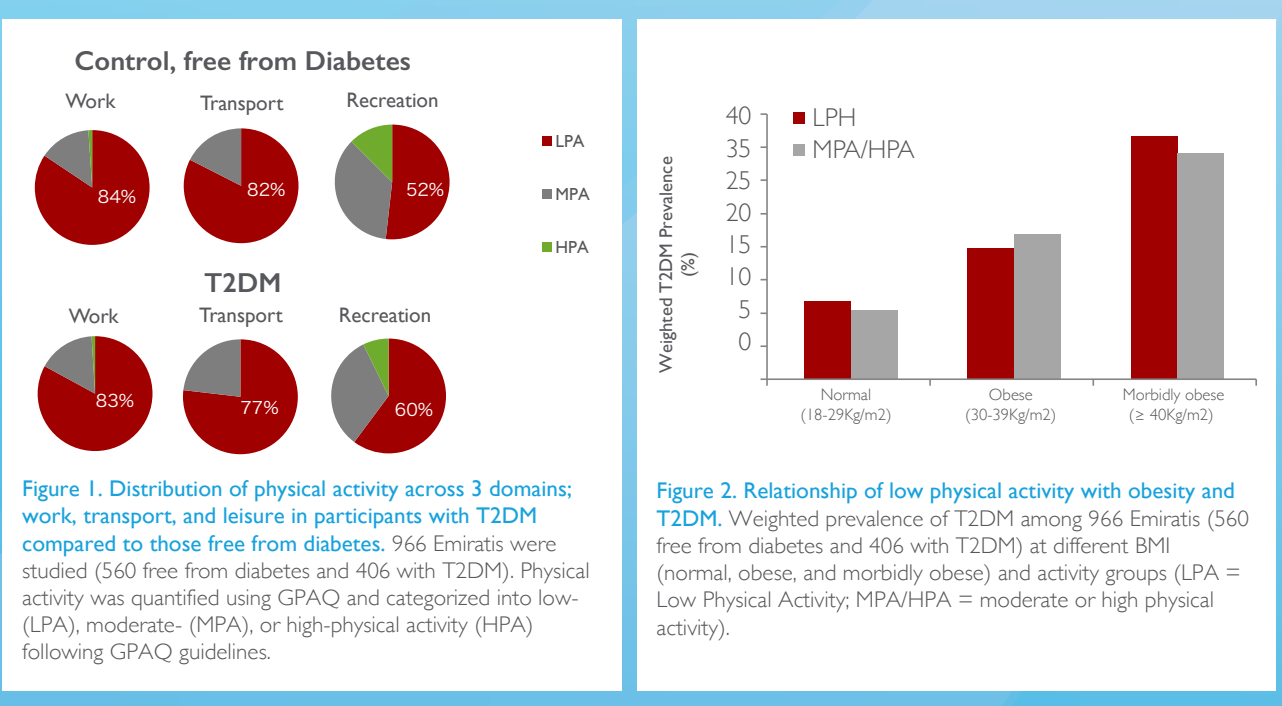

\section{Discussion}

- High rates of LPA regardless of diabetes or obesity status among Emiratis.

- No additive influence of LPA observed on T2DM risk after correction for adiposity.

- Association of LPA with T2DM was not detected in this case-control study.

- Assessing causality of physical inactivity on the development of T2DM will require longitudinal follow-up studies.

\section{Acknowledgements}

This study has been supported by Imperial College London Diabetes Centre 